\section{Occurrence of antibiotic residues in Apulian honey: potential risk of environmental pollution by antibiotics}

\author{
Alessandra Emilia Savarino, ${ }^{1}$ \\ Valentina Terio, ${ }^{1}$ Roberta Barrasso, ${ }^{1}$ \\ Edmondo Ceci, ${ }^{1}$ Sara Panseri, ${ }^{2}$ \\ Luca Maria Chiesa, ${ }^{2}$ \\ Elisabetta Bonerba ${ }^{1}$
}

${ }^{1}$ Department of Veterinary Medicine, University of Bari Aldo Moro, Bari; ${ }^{2}$ Department of Health, Animal Science and Food Safety, University of Milan, Milan, Italy

\begin{abstract}
The presence of antibiotic residues in honey is widely documented and is attributed almost exclusively to improper beekeeping practices, due to the frequent use of drugs for the treatment of beehive diseases. Therefore, the aim of our research was to evaluate the presence of antibiotics in honeycomb using the Anti-Microbial Array II (AM II) and IV (AM IV) method and to assess the relationship between environmental context and antibiotic residues in honey. The results show the presence of antibiotic residues in 26/50 honey from brood nests samples, confirming the impact of environmental contamination on the health quality of this food product. In addition, subsequent analyses conducted on positive samples reveal the instability over time of antimicrobial molecules in honey. These results highlight the need for further studies in order to understand all likely sources of contamination and to implement a comprehensive safety management plan for honey.
\end{abstract}

\section{Introduction}

Honey is a natural food product, much appreciated by consumers for its special functional properties. In Europe, bees are considered food-producing animals and therefore beehive products must comply with legislation on residues of pharmacologically active substances.

Currently, European regulation No. 37/2010 (Commission Regulation (EU) No. 37/2010) has not established MRLs for antimicrobial substances in honey and therefore the use of antibiotics in beekeeping is not allowed in the European community. The absence of MRLs therefore means "zero tolerance" for antibiotic residues in honey, which corresponds to the detection limit of the analytical method used.

Limits of detection may vary depending on the detection method adopted, and there may therefore be different interpretations between European member states. In Italy, the National Residue Control Plan (Italian Ministry of Health, 2017), established in accordance with Council Directive $96 / 23 / \mathrm{EC}$, provides for the detection of chloramphenicol, nitrofuran metabolites, nitroimidazoles, tetracyclines, sulphamides, aminoglycosides and macrolides in order to detect the illegal use of antibiotics in beekeeping. Specifically, the detection limits set by the national plan are $1.3-1.6 \mathrm{~g} / \mathrm{kg}$ for aminoglycosides and $5 \mathrm{~g} / \mathrm{kg}$ for sulphamides, tetracyclines and macrolides

Even though antibiotic drugs are not authorized for the treatment of bees, many studies show the presence of residues in honey, raising the suspicion that this is caused mainly by illegal use in beekeeping. During flight, as bees are exposed to various pollutants dispersed in the environment, the antibiotic contamination could result from their presence in the territory. Indeed, several studies show the presence of pharmacological substances in different environmental compartments such as soil and water (Chung et al., 2017). Currently, there have been only very limited studies examining the relationship between the presence of antibiotic residues and the environmental context in which bees collect food.

In order to understand the potential for drug contamination in honey by environmental antibiotic pollutants, the aim of the present work was to evaluate the presence of antibiotics in honeycomb using the AntiMicrobial Array II (AM II) and IV (AM IV) method, a competitive chemiluminescent assay.

\section{Materials and Methods}

\section{Chemicals and reagents}

All solvents (HPLC/analytical grade) were purchased from Fluka (SigmaAldrich, St. Louis, MO, USA). Trichloroacetic acid (TCA) crystals and the reagents to prepare an EDTA-McIlvaine buffer solution, $\mathrm{pH} 4$ (disodium hydrogen phosphate dihydrate, citric acid monohydrate and EDTA) were purchased from Fluka.

Formic acid (98-100\%) was obtained from Riedel-de Haën (Sigma-Aldrich, St. Louis, MO, USA).

The extraction cartridges (Oasis HLB 3 $\mathrm{mL}, 60 \mathrm{mg}$ ) were provided by Waters (Milford, MA, USA).Amoxicillin, ampi-
Correspondence: Alessandra Emilia Savarino, Department of Veterinary Medicine, University of Bari Aldo Moro, Strada Provinciale 62 per Casamassima Km 3, 70010 Valenzano, Bari, Italy.

Tel.: +39 0805443878; Fax: +390805443855

E-mail: savarino.alessandra@virgilio.it

Key words: Antibiotic residues, honey, environmental pollution, multi-array.

Contributions: The authors contributed equally.

Conflict of interest: The authors declare no potential conflict of interests.

Funding: This research was financially supported by Regione Puglia (Department of Agriculture, Rural Development and Environment - Section of competitiveness of food supply chains): Research and Experimentation Project in Agriculture "Ape e Ambiente: Biomonitoraggio e Valorizzazione dei Prodotti dell'alveare Pugliesi" (AP.A.Bi.Va.P.P.) (Cod PSR 115); Regulation (EU) No 1308/2013 of the European Parliament and of the Council of 17 December 2013, financing of aid applications 2018

Availability of data and materials: The data that support the findings of this study are available from the corresponding author upon reasonable request.

Ethics approval and consent to participate: This research was conducted in accordance with all relevant guidelines and procedures.

Consent for publication: The manuscript does not contain any individual person's data in any form.

Received for publication: 14 November 2019 . Accepted for publication: 21 November 2019.

This work is licensed under a Creative Commons Attribution-NonCommercial 4.0 International License (CC BY-NC 4.0).

(C) Copyright: the Author(s), 2020

Licensee PAGEPress, Italy

Italian Journal of Food Safety 2020; 9:8678

doi:10.4081/ijfs.2020.8678

cillin, benzylpenicillin, cefalexin, cefquinome, ceftiofur, chloramphenicol, chlortetracycline, ciprofloxacin, danofloxacin, dimetridazole, doxycycline, enrofloxacin, florfenicol, florfenicol amine, flumequine, furaltadone, furazolidone, lincomycin, lomefloxacin hydrochloride, marbofloxacin, nalidixic acid, nitrofurazone, oxolinic acid, oxytetracycline, ronidazole, spyramicin, sulphadiazine, sulphadimethoxine, sulphadimidine, sulphamerazine, sulphathiazole, tetracycline hydrochloride, thiamphenicol, tilmicosine, 
tinidazole, trimethoprim, tylosin, and enrofloxacin $\mathrm{d} 5$ as the internal standards (IS) (purity $>98 \%$ ) were used and purchased from Fluka (Sigma-Aldrich, St. Louis, MO, USA).

\section{Standard solutions}

For each standard, stock solutions were prepared $\left(1 \mathrm{mg} \mathrm{mL}^{-1}\right)$ in methanol and kept at $-20^{\circ} \mathrm{C}$. Working solutions at 10 and 100 $\mathrm{ng} \mathrm{mL}^{-1}$ were prepared daily and maintained at $4^{\circ} \mathrm{C}$ during the assay.

\section{Sampling}

Sampling was carried out by taking samples from various areas to obtain information on the impact of different environmental contexts on the quality of honey.

The study included placing a total of 25 hives on the Apulian territory; 15 hives were positioned in five different geographical areas of the province of Bari, of which 3 hives were placed in Toritto, 3 in Conversano, 3 in Castellana Grotte, 3 in Monopoli and 3 in Altamura. In addition, 10 hives were placed at the experimental apiary of the University of Bari A. Moro, Department of Veterinary Medicine.

In total, from the 25 apiaries considered and in which no pharmacological treatment has been carried out, 50 honeycomb samples were taken: 6 from the apiaries of Toritto, 6 from Conversano, 6 from Castellana Grotte, 6 from Monopoli, 6 from Altamura, with another 20 samples from the hives at the experimental apiary of the University of Bari A. Moro. The sampling was carried out according to the guidelines of the National Reference Centre for Beekeeping, which provides for the collection of about $100 \mathrm{~g}$ of honey from brood nests comb without operculum. In short, a portion of the comb $(10 \times 10 \mathrm{~cm})$ was taken with a sterile scalpel and the sample was closed in sterile bags and stored at $-20^{\circ} \mathrm{C}$ until the day of analysis.

\section{Evidence Investigator ${ }^{\mathrm{TM}}$ system}

The honeycomb samples, collected in the year 2018, were tested by Evidence Investigator ${ }^{\mathrm{TM}}$ Anti-Microbial Array II and IV in order to reveal the presence of antibiotics.

\section{Principle}

The Evidence Investigator ${ }^{\mathrm{TM}}$ AntiMicrobial Array II and The Evidence Investigator ${ }^{\mathrm{TM}}$ Anti-Microbial Arrays IV are commercially available kits for the simultaneous quantitative detection of multiple groups of antibiotics. Anti Microbial Array II kit (AM II) is for the simultaneous detection of quinolones, ceftiofur, thiamphenicol, streptomycin, tylosin and tetracyclines.

Anti Microbial Array IV kit (AM IV) is for the detection of groups of aminoglycoside and macrolide antibiotics and specifi- cally detects 12 different types of antibiotics such as amikacin/kanamicin (AMK), apramycin (APA), neomycin/paromomycin (NEO), spectinomycin/dihydrostreptomycin (SPT), streptomycin (STR), tobramycin (TOB), spiramycin (SPR), erythromycin (ERY), tylosin B (TYB), bacitracin (BCT), lincosamides (LIN), and virginiamycin (VIR), simultaneously.

The core technology is the Randox biochip, a solid substrate containing a series of discrete test regions of immobilized antibodies specific for various antimicrobials. A competitive chemiluminescent immunoassay is applied. Increased antimicrobial levels in a sample result in a reduction in the binding of the antimicrobial labelled with horseradish peroxidase (HRP) and thus a decrease in the emitted chemiluminescence signal. The emission of light occurs by chemical reaction, with an enzyme being used to catalyse the chemical reaction on the biochip, which generates the chemiluminescent signal. Luminol and Peroxide are the signal reagents that create a luminous reaction. The light emitted by the chemiluminescent reaction that occurs in each discrete test region (DTR) is simultaneously, detected and quantified by a camera with a charge-coupling device (CCD) at $-50^{\circ} \mathrm{C}$. The CCD camera simultaneously records the light output from all discrete test sites on each biochip on the biochip vector. The concentration of the analytes detected in the sample is calculated from the calibration curve.

\section{Honey sample preparation}

The honey sample ( $1 \mathrm{~g}$ ) was added with $9 \mathrm{~mL}$ of diluted wash buffer warmed to $37^{\circ} \mathrm{C}$. Subsequently, the tubes (Falcon TM Round-Bottom Polypropylene, Fisher Scientific Company, Ottawa, Ontario) containing the samples were placed on a roller for 10 minutes or until dissolution. The preparation was diluted with an equal amount of diluted wash buffer $(1 \mathrm{~mL}+1$ $\mathrm{mL}$ ). After these simple steps, the sample was ready for application to the biochip. The Evidence Investigator ${ }^{\mathrm{TM}}$ analyser does not automatically evaluate the sample dilution; therefore, the results were multiplied by 20 to obtain the final concentration of the sample. For the application of the Honey protocol, the calibrators and the control were reconstituted with the reconstitution buffer provided by the kit.

\section{Calibration and materials}

A nine-point calibration was performed using the AM II and AM IV calibrators, covering the measuring range of all tests. A maximum of 6 carrier biochips can be analysed simultaneously and a new calibration curve is suggested for each series of tests.
All materials were balanced at room temperature and the solutions required for the test were prepared according to the instructions provided. All analyses were carried out in accordance with the manufacturer's recommendations.

\section{Assay protocol}

The procedure for the analysis of samples was as follows: $100 \mathrm{~mL}$ of test dilution solution was inserted into the biochip wells, then $100 \mathrm{~mL}$ of the calibrator or sample was pipetted into the biochip wells. To mix the reagents, all edges of the handling tray were tapped gently. The handling tray was fixed to the base plate of the thermal stirrer and incubated for 30 minutes at $25^{\circ} \mathrm{C}$ and 370 rpm. $100 \mathrm{~mL}$ of the working strength conjugate was pipetted into the biochip wells and the plate was incubated for an additional 60 minutes at $25^{\circ} \mathrm{C}$ and $370 \mathrm{rpm}$. The handling tray containing the media was removed from the thermal stirrer. Reagents were discarded by sharply shaking the handling tray. Two rapid wash cycles were immediately performed, using approximately $350 \mathrm{~mL}$ of wash buffer per well. The handling tray was gently tapped to release any reagents trapped under the biochip. The wash cycle was performed four more times. For each cycle, all four edges of the manipulation plate were gently tapped for approximately 10-15 s; the biochips were then soaked in Wash Buffer for $2 \mathrm{~min}$. After final washing, the wells were filled with Wash Buffer and left to soak until image capture. Shortly before the signal was added, the wash buffer was removed; $250 \mathrm{~mL}$ of the working signal reagent was added to each biochip well and covered to protect it from light. The working signal reagent consisted of Luminol (Luminol-EV 805 for AMII or Luminol-EV841 for AMIV) (1 x $10 \mathrm{~mL})$ and Peroxide $(1 \times 10 \mathrm{~mL})$ mixed in a ratio of $1: 1$. After exactly 2 minutes, the holder was inserted into the Evidence Investigator ${ }^{\mathrm{TM}}$. The captured images and results were processed by a dedicated software application, an integrated graphical user interface and an e-touch software, respectively.

\section{Sensitivity}

The limits of detection (LOD) for the Evidence $^{\mathrm{TM}}$ analytes for the honey matrix is shown in Table 1.

\section{Evaluation of antibiotic depletion}

In order to verify antibiotics depletions in honey, further, where honeycomb samples were detected to be positive, the samples were retested, after approximately 12 months, with Evidence Investigator ${ }^{\mathrm{TM}}$ AMII and AMIV system and confirmatory testing is implemented by LC-Orbitrap method as 
described by Chiesa et al. (2018). During this period, positive samples were stored at $35^{\circ} \mathrm{C}$ in an incubator to simulate the temperature of the beehive, until the time of analysis.

\section{LC-HRMS Orbitrap}

\section{Extraction and clean up}

An aliquot ( $1 \mathrm{~g}$ wet weight) of honey, spiked with the IS at a final $5 \mathrm{ng} \mathrm{mL} \mathrm{m}^{-1}, 100$ $\mu 1$ of $20 \%$ TCA for protein precipitation, and $5 \mathrm{~mL}$ McIlvaine buffer ( $\mathrm{pH} 4.0$ ), were homogenized and were vortexed and sonicated for $15 \mathrm{~min}$. After centrifugation (2500 $\mathrm{g}, 4^{\circ} \mathrm{C}, 10 \mathrm{~min}$ ), the supernatant was transferred to a clean polytetrafluoroethylene centrifuge tube.

The obtained extracts were purified by SPE Oasis HLB cartridges under vacuum.

The SPE cartridges were preconditioned with $3 \mathrm{~mL}$ methanol and $3 \mathrm{~mL}$ Milli$\mathrm{Q}$ water. The samples were loaded, and then washed with $2 \times 3 \mathrm{~mL}$ methanol:water (5:95 $\mathrm{v} / \mathrm{v})$.

Finally, the analytes were eluted with 5 $\mathrm{mL}$ methanol. The eluate was evaporated in a rotary vacuum evaporator at $40^{\circ} \mathrm{C}$.

$200 \mu \mathrm{L}$ methanol:water (10:90 v/v) were uses to reconstitute the dried extract, and then transferred to an auto-sampler vial. The injection volume was $10 \mu \mathrm{L}$.

\section{HPLC-MS/MS analyses}

The simultaneous detection of the antibiotics was performed by LC-Orbitrap.

For the chromatographic separation a were used Surveyor MS quaternary pump with a degasser, a Rheodyne valve with a 20- $\mu \mathrm{L}$ loop and a Surveyor AS autosampler with a column oven (Thermo Fisher Scientific, San Jose, CA, USA), equipped with a Synergi Hydro-RP reverse-phase HPLC column $(150 \times 2.0 \mathrm{~mm}$, i. d. $4 \mu \mathrm{m})$, with a C18 guard column $(4 \times 3.0 \mathrm{~mm}$; Phenomenex, Torrance, CA, USA).

The mobile phase was a binary mixture of aqueous formic acid $0.1 \%$ (solvent $\mathrm{A}$ )

Table 1. Detection limits for the Evidence Investigator ${ }^{\mathrm{TM}} \mathrm{AM}$ IV and for the Evidence Investigator ${ }^{\mathrm{TM}}$ AM II.

\begin{tabular}{lccc} 
& Randox AM IV & \multicolumn{2}{c}{ Randox AM II } \\
Analyte & Honey LOD $(\mathrm{ppb})$ & Analyte & Honey LOD (ppb) \\
Spiramycin (SPR) & 2.0 & Quinolones (QNL) & 3.0 \\
Apramycin (APA) & 2.0 & Ceftiofur (CEFT) & 2.0 \\
\hline Bacitracin (BCT) & 1.2 & Thiamphenicol (TAF) & 1.0 \\
Neomycin (NEO) & 1.0 & Streptomycin (STR) & 5.0 \\
\hline Tobramycin (TOB) & 4.0 & Tylosin (TYL) & 1.0 \\
Tylosin B (TYB) & 1.0 & Tetracycline (TCN) & 5.0 \\
\hline Spectinomycin (SPT) & 2.6 & & \\
Amikacin (AMK) & 6.0 & & \\
\hline Lincosamides (LIN) & 8.0 & & \\
Erytromycin ERY & 2.5 & & \\
\hline Streptomycin (STR) & 4.0 & & \\
Virginiamycin (VIR) & 2.0 & & \\
\hline
\end{tabular}

Table 2. Mean ( \pm sd) antibiotic concentration in samples of honeycomb.

\begin{tabular}{|c|c|c|c|c|c|c|c|c|c|c|c|c|}
\hline & \multicolumn{2}{|c|}{$\begin{array}{l}\text { Toritto } \\
\left(\mathrm{N}^{\mathrm{a}}=6\right)\end{array}$} & \multicolumn{2}{|c|}{$\begin{array}{l}\text { Conversano } \\
\left(\mathrm{N}^{\mathrm{a}}=6\right)\end{array}$} & \multicolumn{2}{|c|}{$\begin{array}{l}\text { Castellana Grotte } \\
\left(\mathrm{N}^{\mathrm{a}}=6\right)\end{array}$} & \multicolumn{2}{|c|}{$\begin{array}{l}\text { Monopoli } \\
\left(\mathrm{N}^{\mathrm{a}}=6\right)\end{array}$} & \multicolumn{2}{|c|}{$\begin{array}{c}\text { Altamura } \\
\left(\mathrm{N}^{\mathrm{a}}=6\right)\end{array}$} & \multicolumn{2}{|c|}{$\begin{array}{l}\text { Experimental Apiary } \\
\left(N^{2}=20\right)\end{array}$} \\
\hline & $\begin{array}{l}\text { Mean } \pm \\
\text { SD }(p p b)\end{array}$ & $n^{b}$ & $\begin{array}{l}\text { Mean } \pm \\
\text { SD }(p p b)\end{array}$ & $\mathrm{n}^{\mathrm{b}}$ & $\begin{array}{l}\text { Mean } \pm \\
\text { SD }(p p b)\end{array}$ & $n^{b}$ & $\begin{array}{l}\text { Mean } \pm \\
\text { SD }(p p b)\end{array}$ & $n^{b}$ & $\begin{array}{l}\text { Mean } \pm \\
\text { SD }(p p b)\end{array}$ & $n^{b}$ & $\begin{array}{l}\text { Mean } \pm \\
\text { SD (ppb) }\end{array}$ & $n^{b}$ \\
\hline QNL & n.d. & - & n.d & - & n.d & - & n.d & - & $9.14 \pm 0.28$ & 6 & n.d & - \\
\hline CEFT & n.d & - & n.d & - & n.d & - & n.d & - & $7.1 \pm 1.77$ & 6 & n.d & - \\
\hline TAF & n.d & - & n.d & - & n.d & - & n.d & - & $1.08 \pm 0.88$ & 4 & n.d & - \\
\hline STR & n.d & - & n.d & - & n.d & - & n.d & - & n.d & - & $23.3 \pm 8.6$ & 20 \\
\hline TYL & n.d & - & n.d & - & n.d & - & n.d & - & n.d & - & n.d & - \\
\hline TCN & n.d & - & n.d & - & n.d & - & n.d & - & n.d & - & n.d & - \\
\hline SPR & n.d & - & n.d & - & n.d & - & n.d & - & n.d & - & n.d & - \\
\hline $\mathrm{APA}$ & n.d & - & n.d & - & n.d & - & n.d & - & $14.3 \pm 1.94$ & 6 & n.d & - \\
\hline BCT & n.d & - & n.d & - & n.d & - & n.d & - & $1.67 \pm 0.37$ & 4 & n.d & - \\
\hline NEO & n.d & - & n.d & - & n.d & - & n.d & - & $1.3 \pm 0.31$ & 3 & n.d & - \\
\hline ТОВ & n.d & - & n.d & - & n.d & - & n.d & - & $10.2 \pm 0.98$ & 5 & n.d & - \\
\hline TYB & n.d & - & n.d & - & n.d & - & n.d & - & n.d & - & n.d & - \\
\hline SPT & n.d & - & n.d & - & n.d & - & n.d & - & $5.1 \pm 2.7$ & 6 & n.d & - \\
\hline AMK & n.d & - & n.d & - & n.d & - & n.d & - & $23.9 \pm 1.3$ & 6 & n.d & - \\
\hline LIN & n.d & - & n.d & - & n.d & - & n.d & - & $30.6 \pm 0.96$ & 6 & n.d & - \\
\hline ERY & n.d & - & n.d & - & n.d & - & n.d & - & $7.3 \pm 0.73$ & 6 & n.d & - \\
\hline VIR & n.d & - & n.d & - & n.d & - & n.d & - & n.d & - & n.d & - \\
\hline
\end{tabular}

aTotal number samples; ${ }^{\mathrm{b} T o t a l}$ number of positive samples; n.d. not detected. 
and methanol (solvent B). The run was performed at $0.3 \mathrm{~mL} \mathrm{~min}-1$ for a total of 25 min: started with $5 \% \mathrm{~B}$, increased to $95 \% \mathrm{~B}$ in $10 \mathrm{~min}$ and remained constant up to the 14th min; then, after an equilibration time of $8 \mathrm{~min}$, the initial conditions were reached again at the 17 th min.

All analytes were detected with a Thermo Q-Exactive Plus Orbitrap (Thermo Scientific, San Jose, CA, USA), equipped with a heated electrospray ionization source. Set in the positive (ESI+) mode, except chloramphenicol, florfenicol and thiamphenicol, which were detected in the negative (ESI-) mode.

The instrumental analytical conditions and validation parameters were the same reported by Chiesa et al. (2018).

Detection of the analytes was based on the calculated exact mass of the protonated/deprotonated molecular ions and at least one specific and typical fragment.

The exact mass of the compounds was calculated using Xcalibur 3.0 software (Thermo Fisher Scientific).

\section{Results}

The Evidence Investigator ${ }^{\mathrm{TM}}$ system is an adequate analytical method for screening analyses for the detection of antibiotics in honey, demonstrating good specificity and accuracy, speed and ease of use (Popa et al., 2012). The results of the analysis performed with Evidence Investigator ${ }^{\mathrm{TM}}$ AntiMicrobial Arrays II and IV during the year 2018 showed the presence of antibiotic residues in 26 of the 50 honey samples analysed. Twenty-four samples showed levels not detectable by the Evidence Investigator ${ }^{\mathrm{TM}}$ Anti-Microbial Arrays II and IV and, therefore, were reported as N. D.

In relation to the geographical position of the hives, only honey samples from the hives located in Altamura and the experimental apiary at Bari University contained residues of antimicrobial drugs.

Briefly, out of fifty honey samples analysed, streptomycin was detected in only 20 samples of honey from the experimental apiary at the University of Bari. Other classes of antibiotics were detected exclusively in honeys from Altamura. The results obtained are shown in detail in Table 2.

The samples that contained antimicrobial residues were retested in the year 2019 with the Evidence Investigator ${ }^{\mathrm{TM}}$ AntiMicrobial Arrays II and IV. The results showed the absence of any of the detectable antibiotic molecules in any honey samples (Table 3). In addition, simultaneous detection of antibiotics was performed by LCOrbitrap on the same honeycomb samples, confirming the results obtained by the screening method adopted.

\section{Discussion and Conclusions}

Food safety is a priority to be pursued all over the world and the presence of chemical substances in food is a widespread problem. Antimicrobial drugs in honey are a serious health risk as they can cause toxic, allergic and hypersensitivity reactions (Reybroeck, 2014); in addition, they can promote the spread of resistant microbial strains (Wegener et al., 1999). Different studies have shown the presence of antibiotics in European and non-EU honey (Saridaki-Papakonstadinou et al., 2006; Baggio et al., 2009; Barrasso et al., 2018) and, in some cases, mixing with Chinese honeys has been thought to be the reason for the findings of streptomycin, tetracycline and chloramphenicol in European honey (Reybroeck, 2003).

According to the scientific bibliography, improper beekeeping practices are responsible for antibiotics in honey and in beehive products. Our research is therefore one of the first studies that relates the presence of chemotherapeutic residues to the environmental production context and highlights the possible impact of agricultural activities on the health quality of honey. The locations of the hives are characterized by different agricultural practices. The honeys contaminated with antibiotics came from areas where animal husbandry is

Table 3. Comparison of the antibiotic concentrations detected in the year 2018 and in the year 2019.

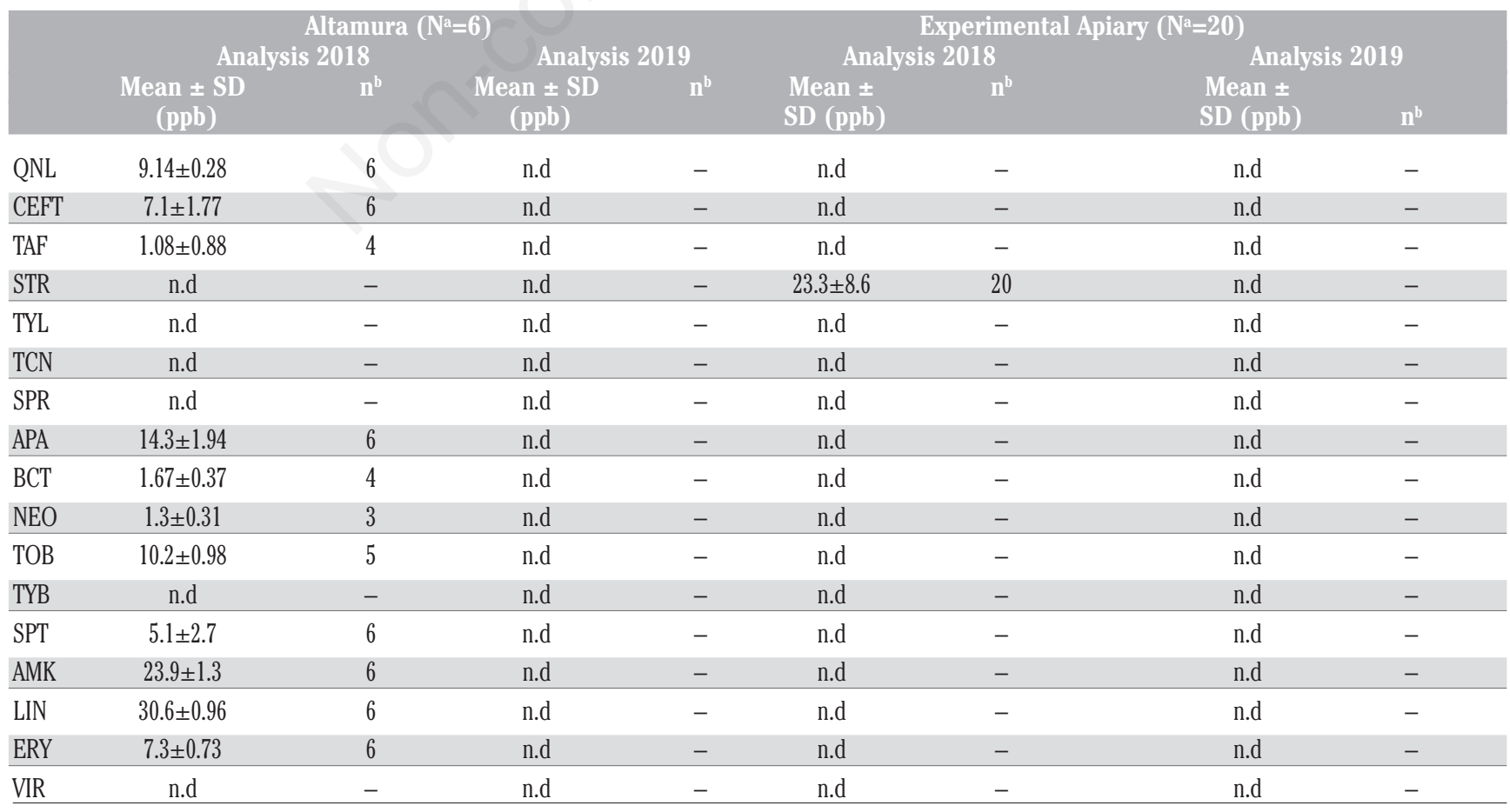

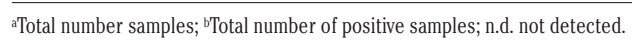


widespread. In the other geographical areas assessed, livestock farming is uncommon.

Different classes of antibiotics were detected in honeys from Altamura and indeed, according to the latest censuses reported in the database of the National Livestock Register, this geographical area holds numerous livestock farms with various species of animal. In honey samples from this area, some antibiotics were found at high concentrations; in particular, amikacin concentrations were in the range of 22.48 to $26.15 \mathrm{ppb}$, while lincosamide contents ranged between 29.45 and 31.65 ppb. Both substances are widely used to treat bacterial disease and some protozoa in cattle, sheep and pigs. The presence of streptomycin in honeycomb from the experimental apiary at the University of Bari probably originates from local sheep farms, where it is used to treat ovine mastitis.

The pharmacological substances investigated in our study are widely used in veterinary medicine which makes environmental contamination likely. Antibiotics are administered in different ways in animal husbandry for therapeutic or preventive purposes, thus contributing to their spread in the environment (Chung et al., 2017). Some drugs are eliminated via faeces, urine, and spreading manure from treated animals increases their distribution in the environment (Broekaert et al., 2011). Several studies have shown that plants can absorb drugs from the soil, which then spread to different plant compartments. The presence of some classes of antibiotics such as tetracyclines, sulfonamides, enrofloxacin, amoxicillin and tylosin in such plant products for human consumption as lettuce, carrots, tomatoes, vetch and maize has been documented (Ahmed et al. , 2015).

The uptake of drugs and their distribution in various vegetable compartments is conditioned by many factors, such as the species and physiological phase of the plant, soil characteristics, and the chemical composition and concentration of the drug (Chung et al., 2017). In addition, environmental studies also show the presence of antibiotics in deep, waste, surface (Kolpin et al., 2002) and drinking waters (Barnes et al., 2008).

Some drugs such as sulfanimide and tetracycline are administered in drinking water on farms, so water can become a source of pollution for hive products. The bee explores territories even $6 \mathrm{~km}$ away from the hive in order to forage food and water or take honey from other apiaries and by doing so they contaminate their own.

The results of the present study show that honey fresh from the hive may contain various residues of antimicrobial sub- stances. However, the presence of residues was not confirmed by tests carried out more than one year after sampling. The results of the analysis conducted by Evidence Investigator ${ }^{\mathrm{TM}}$ Anti-Microbial Arrays II and IV and LC-Orbitrap at a distance of months showed the absence of antibiotics in honeycomb positive at the first screening assay. The failure to detect any antibiotic substance in our samples could be caused by the degradation of drugs in honey. Many studies have shown the degradation of antimicrobials in acid media such as honey (Kochansky, 2004; Thompson et al., 2007; Thompson and Van de Heveer, 2012). Some pharmacological compounds remain stable in honey, while other compounds are modified to yield a variety of by-products (Thompson and Van de Heveer, 2012).

The stability of beta-lactams and quinolones have been studied in muscles and frozen stock solutions. Quinolones were stable for about 3 months in chicken muscle kept at $-20^{\circ} \mathrm{C}$, however this result cannot be extended to honey (Bailac et al., 2006). Unfortunately, few data on the stability of beta-lactamic and quinolone antibiotics and in honey are available in the literature. However, concerning fluoroquinolones, one study showed a reduction in ciprofloxacin concentrations from values of $>10 \mathrm{mg} \mathrm{kg}^{-1}$ to $622-1370 \mu \mathrm{g} \mathrm{kg}^{-1}$ after 18 weeks (Fussell et al., 2010). The study mentioned could support the results of our investigation, considering that the highest initial value of quinolones we found was $0.00965 \mu \mathrm{g} \mathrm{kg}^{-1}$, significantly lower than the initial values reported by Fussell et al. (2010), and the reduction in concentration was determined after a longer period. Regarding thiamphenicol (TAP) and florfenicol (FFC) in honey, no data are available. Their stability has been assessed in fish samples stored at $-18^{\circ} \mathrm{C}$ in the absence of light (Evaggelopoulou and Samanidou, 2013); both antibiotics showed stability for two weeks.

The stability of streptomycin is considered to be about four or five months in honey stored at room temperature (Pang et al., 2004; Anon, 2006), whereas for lincomycin, this period appears to be 28 days under the same temperature conditions. However, Adams et al. (2009) showed that after treatment with $1.2 \mathrm{~g}$ lincomycin hydrochloride per hive, antibiotic was persistent in the hive 290 days after dosing. Alippi et al. (1999) showed that a period of 35 to 40 days after administration of erythromycin is required to obtain residue levels below the limit of detection $\left(50 \mu \mathrm{g} \mathrm{kg}^{-1}\right)$. In addition, under acidic conditions, erythromycin degrades to form by-products (Volmer \& Hui, 1998) with no antimicrobial activity but suspected to cause gastrointestinal disorders (Pariza, 2006). In agreement with previous studies carried out on honey (Anon., 2002; Kochansky 2004; Martel et al., 2006; Adams et al., 2009; Thompson \& van den Heever, 2012), this study confirms the degradation of antibiotic molecules over time, probably caused by the acidity of the honey. In addition to the chemical degradation of antibiotics in honey, as reported by Reybroeck et al. (2012) a factor influencing the presence of residues in honey at harvest time is the yield of the honey, i.e. the dilution effect. It is affected by weather conditions during flowering and by the geographical context of production. In fact, the highest concentrations of residues in honey are detected within one week of treatment (Reybroeck et al., 2012). Subsequently, the concentration of residues in the honey decreases by a dilution effect of honey flow or degradation of the active compound in other metabolites (Reybroeck et al. 2012).

Considering that the degradation of antibiotics in our honey samples was evaluated after a period of about 365 days after the first screening analysis had been performed, the negative results may be attributed to the above factors.

In summary, our study demonstrates the relationship between the presence of antibiotics in honey and the related production areas involved in intensive breeding, thus excluding improper beekeeping practices. Moreover, our research confirms that bees and beehive products are excellent bio-indicators for environmental monitoring. Honey is a product that is greatly influenced by the environment and, consequently, its contamination might be caused by many factors. Our results show that the location of apiaries in areas far away from husbandry activities reduces the risk of contaminants in honey and therefore this approach may be useful to beekeepers. The presence of contaminants in honey is constantly increasing due to environmental pollution and new agricultural practices and represents a serious risk to consumer health. As several studies have found different chemical contaminants in honey, consumers may be exposed to several chemicals at a time.

In conclusion, understanding the source of pollution is important to preserve the quality of the product and to ensure its safety; moreover, in relation to the high presence of antibiotic residues in honey, the application of rapid, simple screening methods for the detection of multiple analytes is essential.

Considering the lack of studies and the chemical complexity of honey, further analyses will have to be performed and proper monitoring plans and safety management 
systems implemented with the aim both of correctly determining the kinetics of drug degradation in honey and of obtaining a safe and healthy product for human beings.

\section{References}

Adams SJ, Fusell RJ, Dickinson M, Wilkins S, Sharman M, 2009. Study of the depletion of lincomycin residues in honey extracted from treated honeybee (Apis mellifera L.) colonies and the effect of the shook swarm procedure. Anal Chim Acta 637:315-20.

Alippi AM, Albo GN, Leniz D, Rivera I, Zanelli ML, Roca AE, 1999. Comparative study of tylosin, erythromycin and oxytetracycline to control American foulbrood of honey bees. J Apic Res 38:149-58.

Ahmed MB, Rajapaksha AU, Lim JE, Vu NT, Kim IS, Kang HM, Lee SS, Ok YS, 2015. Distribution and accumulative pattern of tetracyclines and sulfonamides in edible vegetables of cucumber, tomato, and lettuce. J Agric Food Chem 63:398-405.

Anon, 2002. Establishing the appropriate treatment method for oxytetracycline to minimise effects on brood and residues in honey. Final project report. Central Science Laboratory, York, United Kingdom.

Anon, 2006. Investigation of the fate of veterinary drugs used in apiculture. Research project final report. Central Science Laboratory, York, United Kingdom.

Baggio A, Gallina A, Benetti C, Mutinelli F, 2009. Residues of antibacterial drugs in honey from the Italian market. Food Addit Contam B 2:52-8.

Bailac S, Barrón D, Barbosa J, 2006. New extraction procedure to improve the determination of quinolones in poultry muscle by liquid chromatography with ultraviolet and mass spectrometric detection. Anal Chim Acta 580:163-9.

Barnes KK, Kolpin DW, Furlong ET, Zaugg SD, Meyer MT, Barber LB, 2008. A national reconnaissance of pharmaceuticals and other organic wastewater contaminants in the United States e I. Groundwater. Sci Total Envir 402:192200.

Barrasso R, Bonerba E, Savarino AE, Ceci E., Bozzo G, Tantillo G, 2019. Simultaneous Quantitative Detection of Six Families of Antibiotics in Honey Using A Biochip Multi-Array Technology. Vet Sci 6:1.

Broekaert N, Vanpeteghem C, Daeseleire E,
Sticker D, Vanpoucke C, 2011. Development and validation of an UPLC-MS/MS method for the determination of ionophoric and synthetic coccidiostats in vegetables. Anal Bioanal Chem 401:3335-44.

Chiesa LM, Panseri S, Nobile M, Ceriani F, Arioli F, 2018. Distribution of POPs, pesticides and antibiotic residues in organic honeys from different production areas. Food Addit Contam A 35:1340-55.

Chung HS, Lee YJ, Rahman MM, Abd ElAty AM, Lee HS, Park BJ, Kim JE, Hacımüftüoğlu F, Nahar N, Shin HC, Shim JH, 2017. Uptake of the veterinary antibiotics chlortetracycline, enrofloxacin, and sulphathiazole from soil by radish. Sci Total Environ 605606:322-31.

Commission regulation (EU) No 37/2010 of 22 December 2009 on pharmacologically active substances and their classification regarding maximum residue limits in foodstuffs of animal origin. Off $\mathrm{J}$ EU 2009, L15, 72.

Evaggelopoulou EN, Samanidou VF, 2013. Development and validation of an HPLC method for the determination of six penicillin and three amphenicol antibiotics in gilthead seabream (Sparus Aurata) tissue according to the European Union Decision 2002/ 657/EC. Food Chem 136:1322-29.

Fussell RJ, Dickinson M, Heinrich K, Wilkins S, Sharman M, 2010. A study on the distribution of veterinary drug residues in treated bee hives and implications for setting MRLs. In: Proceedings of the sixth international symposium on hormone and veterinary drug residue analysis, June 1-4, Gent, Belgium.

Italian Ministry of Health. Direzione Generale per L'igiene e la Sicurezza Degli Alimenti e la Nutrizione. Piano Nazionale Residui 2017. Available online: http://webcache.googleusercontent.com/search?q=cache:w4SXIP28Yc YJ:repository.regione.veneto.it/public/c ed34a0eddc56151da31f57ff969d50c.ph $\mathrm{p} \% 3$ Flang\%3Dit $\% 26 \mathrm{dl} \% 3$ Dtrue $+\& \mathrm{~cd}=$ $1 \& \mathrm{hl}=\mathrm{it} \& \mathrm{ct}=\mathrm{clnk} \& \mathrm{gl}=\mathrm{it}$ (accessed on 12 March 2018).

Kochansky J, 2004. Degradation of tylosin residues in honey. J Apicult Res 43:6568.

Kolpin DW, Furlong ET, Meyer MT, Thurman EM, Zaugg SD, Barber LB, Buxton HT, 2002. Pharmaceuticals, hormones, and other organic wastewater contaminants in US streams, 19992000: a national reconnaissance.
Environ Sci Technol 36:1202-11.

Martel AC, Zeggane S, Drajnudel P, Faucon JP, Aubert M, 2006. Tetracycline residues in honey after hive treatment. Food Addit Contam A 23:265-73.

Pang GF, Zhang JJ, Cao YZ, Fan CL, Lin XM, Li ZY, Jia GQ, 2004. Evaluation of analyte stability and method ruggedness in the determination of streptomycin residues in honey by liquid chromatography with post-column derivatization. J AOAC Int 87:39-44.

Pariza RJ, 2006. From patent to prescription: paving the perilous path to profit. In M. S. Chorghade (Ed.), Drug discovery and development. Drug discovery (Vol. 1, pp. 1-16). Hoboken, NJ: John Wiley \& Sons, Inc.

Popa ID, Schiriac EC, Cuciureanu R, 2012. Multi-analytic detection of antibiotic residues in honey using a multiplexing biochip assay. Rev Med Chir Soc Med Nat Iasi 116:324-329.

Reybroeck W, 2014. Quality control of honey and bee products. In R.K. Gupta, W. Reybroeck, J.W. van Veen, \& A. Gupta (Eds.), Beekeeping for poverty alleviation and livelihood security. Vol. 1: Technological aspects of beekeeping (pp. 481-506). Dordrecht: Springer.

Reybroeck W, Daeseleire E, De Brabander HF, Herman L, 2012. Antimicrobials in beekeeping. Vet Microbiol 158:1-11.

Reybroeck W, 2003. Residues of antibiotics and sulphonamides in honey on the Belgian market. Apiacta 38:23-30.

Saridaki-Papakonstadinou M, Andredakis S, Burriel A, Tsachev I, 2006. Determination of tetracycline residues in Greek honey. Trakia J Sci 4:33-36.

Thompson TS, van den Heever JP, 2012. Degradation of erythromycin in honey and selection of suitable marker residues for food safety analysis. Food Chem 133:1510-20.

Thompson TS, Pernal SF, Noot DK, Melathopoulos AP, van den Heever JP, 2007. Degradation of incurred tylosin to desmycosin-Implications for residue analysis of honey. Anal Chim Acta 586:304-11.

Volmer DA, Hui JPM, 1998. Study of erythromycin A decomposition products in aqueous solution by solid-phase microextraction/liquid chromatography/tandem mass spectrometry. Rapid Commun Mass Spectrom 12:123-9.

Wegener HC, Aarestrup FM, Gerner-Smidt P, Bager F, 1999. Transfer of antibiotic resistant bacteria from animals to man. Acta Vet Scand 92:51-7. 